\title{
Enhanced Interference Cancellation to Mitigate the CRS Interference in LTE/LTE-A Heterogeneous Networks
}

\author{
S. Shibu ${ }^{1}$, V. Saminadan ${ }^{2}$ \\ ${ }^{1}$ Research Scholar, ${ }^{2}$ Professor \\ ${ }^{1,2}$ Department of Electronics and Communication Engineering, \\ Pondicherry Engineering College, Puducherry - 605014, India. \\ ${ }^{1}$ soman.shibu@gmail.com \\ ${ }^{2}$ saminadan@pec.edu
}

\begin{abstract}
In the recent years, heterogeneous network (HetNet) is used to improve the spectral efficiency and network capacity of Long Term Evolution-Advanced (LTE-A). Although, it has huge traffic congestion due to numerous numbers of mobile users and data terminals, leads to problems of cooperation between Macro-cell and Pico-cells. One of the major interference sources in LTE-A is Common Reference Signal (CRS) which is transmitted by neighboring cells across the entire frequency band. There are two categories of CRS interference, such as non-colliding CRS and colliding CRS interference. So, these type CRS interferences become the restricting factor for achieving higher throughput in downlink LTE-A. In this paper, an efficient equalizer for mitigating non-colliding CRS and Enhanced Space Alternating Generalized Expectation-Maximization (ESAGE) with a maximum aposteriori (MAP) criterion for colliding CRS interference cancellation is used. The simulation outcomes show that the proposed efficient equalizer and ESAGE-MAP provides better CRS interference cancellation than existing techniques.
\end{abstract}

Keywords: Heterogeneous Network (HetNet), Long Term Evolution- Advanced(LTE-A), Common Reference Signal (CRS).Enhanced Space Alternating Generalized Expectation-Maximization (ESAGE).

\section{INTRODUCTION}

Nowadays, Long Term Evolution (LTE) is a guideline for high-speed wireless communication mainly in mobile phones and data terminals [1]. The LTE system provides many benefits that include speed, efficiency bandwidth utility, etc.. One of the major problems in wireless networks is the restriction of frequency spectrum [2]. Spectral efficiency is reaching its limits; it is essential to increase the node density, which can improve the performance of network capacity. The macro cell deployments are inadequate, when adding another cell it does not produce significant inter-cell interference and cell splitting gain can be performed. However, the dense macro cells are considerably decreased the cell splitting gains because of inter-cell interference [3]. So, another low power node can be deployed instead of deploying macro cell where the low power nodes are known as Pico cell and Femto cell. Hence, the network consists of Macro cell and low power nodes known as heterogeneous network. In a heterogeneous network (HetNet) Pico/Femto cell are provided with the same set of frequencies within the Macro cell, creating more traffic [4]. This heterogeneous network increases the data rate, overall network capacity and the performance of the cell-edge user and also has inter-cell interference in MacroPico/Femto cell deployments. Let's take an example, when the UE is in cell extension region and it is connected to Femto base station. But, the Macro base station UE's received power is much higher than the power received from Femto base station. This power difference causes severe intrusive to the Femto user by Macro base station.

In order to mitigate Inter-cell interference in LTE, 3GPP issue 8 standardization proposed inter-cell interference coordination (ICIC) was used. This is one of the new radio resource management (RRM) functions. The objective of ICIC is to regulate the resources such as frequency, time and power available in both cells and schedule these resources to the users [5]. The coordination between neighboring cells depends on the exchange of interference information. The major problem of ICIC is that it can able to control the data channel interference, but control channels are still under interference.

In LTE-A release 10 introduced enhanced inter-cell interference coordination (eICIC) to overcome interference problems in HetNet [6]. Also eICIC alleviates interference with traffic and control channels. It uses frequency, time and power domain to reduce intra-frequency interference in heterogeneous networks (Hetnet). It has two methods one is Time domain multiplexing inter-cell interference coordination approach called almost blank sub-frame (ABS) and other is a frequency domain multiplexing inter-cell interference coordination 
scheme. ABS is a sub-frame with reduced downlink transmission power and activity. In eICIC, it increases the throughput of Macro cell and degrades the throughput of Femto cell.

Recently, LTE-A release 11 introduced more improved inter- cell interference coordination (FeICIC) [7]. In FeICIC, reduced power ABS was used where the data on PDSCH can be transmitted, but on the reduced power level.

In a heterogeneous network (HetNet), a macro cell transmits ABS with CRS and a Pico cell is transmitting a sub-frame with CRS and also with PDSCH [8]. Hence, CRS spread the sub-frame in both time and frequency domain. These involve a collision between CRS of Pico sub-frame and CRS of ABS which is the worst scenario in heterogeneous network (HetNet). This is called colliding CRS. The CRS is employed for estimation of the channel, if they collide, the quality of the channel estimation and system performance will degrade. The interference effect from PCH, PBCH and PSS/SSS in ABS can be still restricted by not assigning sub-frames where these channel show ABS and also shift between the macro and Femto cell. This is called noncolliding CRS.

This paper describes prior work related to this field in Section 2, followed by 3 presents the proposed technique in detail and the results of simulation are given in Section 4. Section 5 presents the conclusions.

\section{RELATED WORK}

The mobile device users have increased rapidly due to the advancement in technology, so that the network is more congested as well as to reduce the network performance, speed and efficiency of the LTE system. Also, it causes interference between cells, which reduce the performance. To increase LTE system performance with minimal interference, different interference cancellation methods were used.

An enhanced CRS interference cancellation receiver for LTE-A network is proposed [9]. The main idea of this scheme is to produce a soft evaluation of the useful signal using the turbo decoding and cancels this from the overall received signal prior to the approximation of the interfering CRS channels. In this link-level evaluation, results demonstrated that this approach achieves performance gains compared to conventional CRSIC methods and performance close to the ideal CRS-IC receiver.

An IC algorithm that involves an interference signal reconstruction in HetNet receiver is proposed in [10]. At first, the Time Offset / Carrier Frequency Offset (TO/CFO) compensation and interfering channel estimation is applied to reconstructing the interfering CRS signals. Finally, the interference is cancelled in time domain, then recreated by subtracting it from receiving signals. The simulation outcomes had shown that the performances of the IC algorithm achieve better performance in different channel conditions.

CRS-IC for downlink co-channel macro and Pico-eNBs using RE and TDM eICIC is analyzed [11]. In this, RSRP-based CRS-IC method is introduced and evaluated. The simulation result shows that the method achieves similar performance as a fixed scheme cancels a constant number of macro-cells. However, RSRP based CRS- IC has the benefit of not requiring extra inter-eNB signaling/eNB to UE signaling, since the decision on the number of cancelled cells remains on the UE. Also, this scheme offers cell-edge users, which are the ones, intended to use an ABS for data transmissions. Finally, the significant gains are obtained with TDM eICIC which increase throughput up to $85 \%$.

A UE receiver with robust interference mitigation capabilities with the robust equalization by grouping the interfered resource elements and performing equalization jointly for the non-colliding CRS type is proposed [12]. Although the performance of the proposed robust equalizer is similar to that of the traditional CRS-IC type, it has lower complexity and delay. Also, the SAGE-MAP algorithm is introduced for the colliding CRS. Simulation results offer the schemes to provide excellent results. In a harsh Fe-ICIC test scenario, where the UE is interfered by two strong interfering cells, the algorithms can still effectively alleviate the interference.

The advanced interference mitigation techniques for the heterogeneous network (HetNet) deployment of 3GPP LTE-A is analyzed in [13]. In eICIC without proper interference mitigation leads to fail in case of interference scenario of macro-Femto cell with colliding CRSs. To overcome the drawback, the received-power dependent interference cancelation consisting of the direct IC and joint channel estimation, the decision-directed channel estimation (DDCE) and IC-assisted DDCE to improve the channel estimation accuracy under different interference have been investigated.

A low complexity and robust measurement algorithm for LTE is proposed in [14]. This algorithm is capable of cancelling interference from neighboring base stations. The experimental results demonstrated its excellent performance through extensive simulations in test conditions as specified in 3GPP. The measurement accuracy is below $2 \mathrm{~dB}$ for almost all tested cell combinations.

A novel SAGE based Turbo detector is proposed to build up an iterative receiver structure together with synchronization and channel estimation for uplink MIMO-OFDMA systems [15]. In SAGE algorithm, the expectation step intends to remove the Multiple Access Interference (MAI) caused by other asynchronous users. Then the maximization step is utilized to estimate the required parameters sequentially for each user. Simulation 
results show that the proposed SAGE algorithm has lower BLER than the other EM-based detectors with quite a reasonable complexity.

The evolution of inter-cell interference management techniques is thoroughly examined from TDM based enhanced inter-cell interference coordination (eICIC) techniques [16]. It has been used for more advanced Coordinated Multi-Point (CoMP) transmission/reception, while comparing and contrasting their common features and differences. Finally, Carrier Aggregation (CA) is explores in detail as an advanced and challenging mechanism in order to have an efficient resource allocation and inter-cell interference mitigation in LTEAdvanced HetNet environments.

\section{PROPOSED METHODOLOGY}

Macro-Pico HetNet deployment to cell extension region (CRE) is one of the known operations with harsh interference. This operation is shown in Fig. 1. User1 and User2 are served by the relatively close macro and Pico cells respectively. User3 is located within the CRE region of the Pico-cell and is served by it with the purpose to perform macro-cell traffic off-loading and reduce the handover rate of the users by biasing the received signal power for handover decision. This produces severe interference to User3 in addition to a weak signal coming from the serving Pico-cell.

In order to diminish the amount of interference to User3, the interfering macro-cell will transmit an ABS subframe when User3 is scheduled for data transmission by its serving Pico-cell. The coordination between eNBs can be realized by the e-ICIC mechanism and communication among cells is dealt through the $\mathrm{X} 2$ signaling protocol. As shown in Fig. 1, User3 is allocated subframes 2 and 10 by the Pico-cell while the macro-cell is transmitted ABS on those particular subframes.

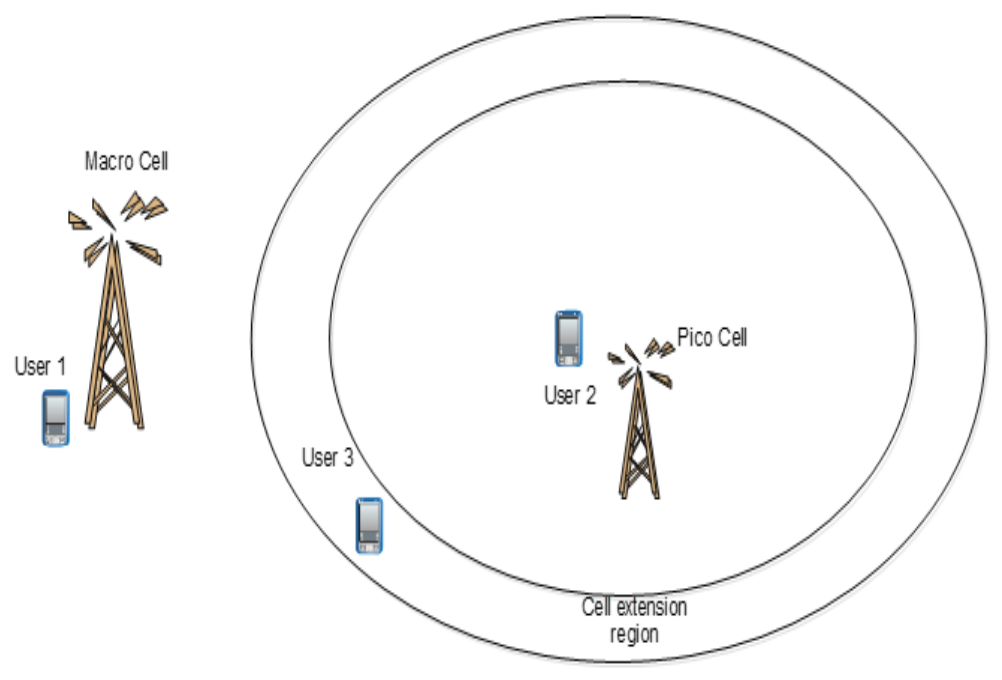

Fig.1 HetNet with Macro-Pico deployment.

Fig.2 Block diagram of LTE-A downlink system. For LTE-A downlink, after cyclic prefix removal the received signal is represented as $\mathrm{y}_{\mathrm{i}}(\mathrm{n})$ for the $\mathrm{i}^{\text {th }}$ symbol can be shown as:

$$
y_{i}(\mathrm{n})=y_{i}^{(\mathrm{m})}(\mathrm{n})+y_{i}^{(\mathrm{p})}(\mathrm{n})+n_{i}
$$

Where $y_{i}^{(\mathrm{m})}(\mathrm{n})$ is a desired signal, $y_{i}^{(\mathrm{p})}(\mathrm{n})$ is an interference signal and $n_{i}$ denotes an additive Gaussian noise.

The fast Fourier transform is performed to transfer the signal into OFDM symbols. After N-point FFT, the OFDM symbol is expressed as:

$$
\begin{gathered}
Y_{i, k}=Y_{i, k}^{(\mathrm{p})}+Y_{i, k}^{(\mathrm{m})}+N_{i, k} \\
Y_{i, k}=H_{i, k}^{(\mathrm{p})} X_{i, k}^{(\mathrm{p})}+\sum_{n=-N / 2}^{N / 2} e^{(2 \pi \mathrm{n} \Delta \mathrm{n} / \mathrm{N})} H_{i, k}^{(\mathrm{m})} X_{i, k}^{(\mathrm{m})} \varphi_{i}+N_{i, k}
\end{gathered}
$$


Where $X_{i, k}^{(\mathrm{p})}$ and $X_{i, k}^{(\mathrm{m})}$ represent the $\mathrm{i}^{\text {th }}$ symbol at $\mathrm{k}^{\text {th }}$ subcarrier for desired signal and interference, respectively; $H_{i, k}^{(\mathrm{p})}$ and $H_{i, k}^{(\mathrm{m})}$ are the channel coefficients of the serving and the interfering channel at $\mathrm{k}^{\text {th }}$ subcarrier, respectively; $\varphi_{i}$ stands for the inter-carrier interference, which arises from CFO; and $\Delta n$ stands for the relative TO between the interfering cell and serving cell.

The channel estimation is evaluated for colliding and non-colliding CRS interference cancellation using an efficient equalizer and ESAGE algorithm respectively.

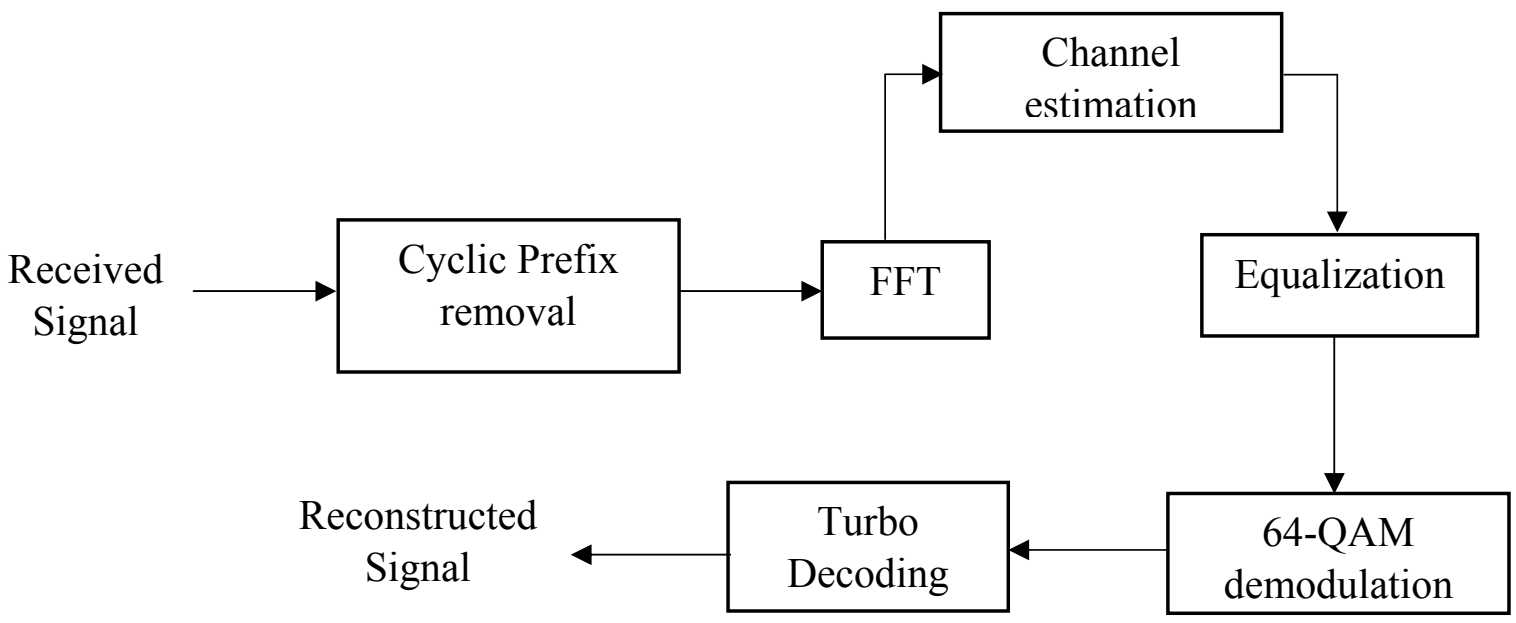

Fig.2 Block diagram of LTE-A downlink system.

\section{A) Efficient equalizer for the Non-Colliding CRS Interference Cancellation}

This proposed paper uses an efficient equalizer for mitigating the non-colliding CRS type of interference. In a conventional receiver, every resource element (RE) carrying data are extracted and then passed to the MIMO detection. In the proposed receiver, every REs carrying data are divided into double sets, namely REs interfered by neighbor cell CRS and the remaining REs carrying data. The symbol detections for the two sets are done separately. In order to describe the concept behind the proposed efficient equalizer, consider the $2 \times 2$ space frequency block-coded (SFBC) modulation as a transmission scheme; however the efficient equalizer can easily be provided to other transmission schemes. For SFBC, the first-set also contains the adjacent RE in the frequency-domain due to the SFBC structure.

The first-set is equalized by the proposed robust equalizer while the second-set is equalized by an ordinary MIMO detector. The LS estimate of the channel with LMMSE equalizer is designed. The LS channel estimate derived from the pilot symbols and only evaluations to be performed in real time is multiplied with the received signal. There is no frequency offset introduced in the simulation of the proposed system. Under the assumption that the user is perfectly synchronized with the serving and the neighbor cells, the FFT received complex signal for the $k^{\text {th }}$ subcarrier, the $l^{\text {th }}$ OFDM symbol within a subframe and at the $i^{\text {th }}$ receive antenna of a user,

$$
Y_{i}[\mathrm{k}, 1]=\sum_{m=1}^{M} \sum_{n=1}^{N} H_{i, j}^{(\mathrm{m})}[\mathrm{k}, 1] \mathrm{X}_{j}^{(\mathrm{m})}[\mathrm{k}, 1]+n_{i}
$$

Where $\mathrm{X}_{j}^{(\mathrm{m})}[\mathrm{k}, 1]$ symbols correspond to either CRS or precoded data transmitted from the $\mathrm{m}$-th cell. The channel frequency response (CFR) of the $\mathrm{m}^{\text {th }}$ cell corresponding to the $\mathrm{i}^{\text {th }}$ receive antenna and the $\mathrm{j}^{\text {th }}$ transmit antenna port is described by $H_{i, j}^{(\mathrm{m})}[\mathrm{k}, 1]$. The complex white Gaussian noise of the $\mathrm{i}^{\text {th }}$ receive antenna is denoted by $n_{i}$ with average noise power $\sigma_{i}^{2}$.

The received RE pair in $2 \times 2$ MIMO SFBC and interference is located in a $\mathrm{k}^{\text {th }}$ sub-carrier, can be expressed in equation (5) as follows: 


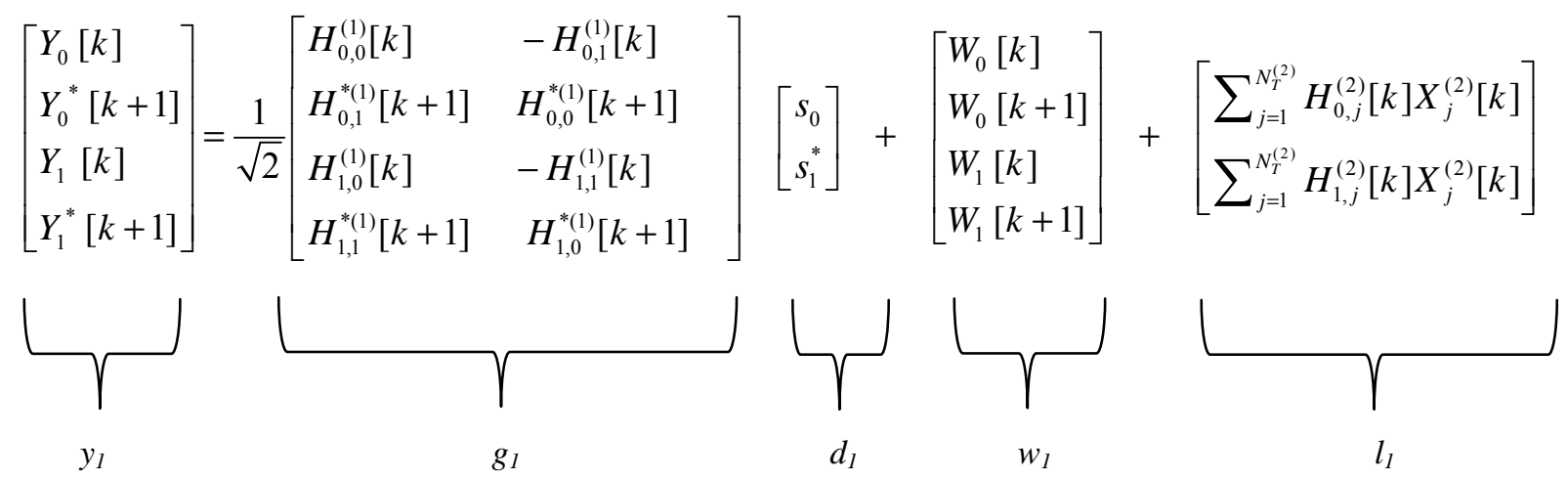

Utilizing the SFBC structure, the received symbols are reorganized such that the set of the symbols is mapped to the $\mathrm{d} 1$. Considering the signal representation from one RE pair is extended to form a group of $\mathrm{N}$ resource element pairs can be given as:

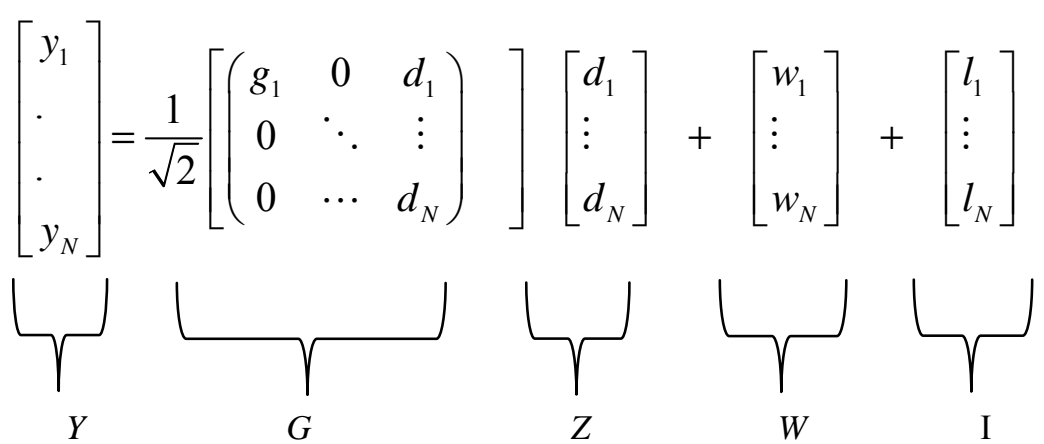

The efficient equalizer performs the joint equalization using a group of $\mathrm{N} R E$ pairs experiencing almost the same channel state. Since the interference is correlated in time and frequency, an equalization in the LMMSE criterion is optimal.

\section{B) ESAGE-MAP algorithm for the Colliding CRS Interference Cancellation}

With the multicellular channel estimation problem in hand, we employ the SAGE algorithm which resolves the multi-cell channel estimation problem into single-cell channel estimation problems. Furthermore, not only CRS-IC is performed implicitly by the considered SAGE framework, but also the accurate channel estimates can be obtained for the SC and the NCs by employing MAP/LMMSE criterion on the decomposed single-cell channel estimation problem.

The matrix inversion causes a prohibitive complexity in the channel estimate, particularly for the multiple antenna conditions. This algorithm splits the high-dimensional problem into many low-dimensional subproblems by conditioning on the parameter subset.

i. Expectation Phase : The $\mathrm{k}^{\text {th }}$ user received signal is divided into the desired signal $y_{p}$ and interference signal $y_{q}$.

$$
\begin{aligned}
& y_{p=} \psi+\beta_{p} e \\
& y_{q=} \sum_{n=1, n \neq k}^{k} \psi+\beta_{p} e
\end{aligned}
$$

The above two equations represents the complete observation $y_{p}$ and $y_{q}$ got from the incomplete observation y. From the desired signal $y_{p}$, evaluate the three parameters $\varepsilon, \mu$, and $\mathrm{t}$. Consider that all users, transmits $\mathrm{N}_{0}$ identical OFDM symbols to the base station.

The expectation phase at the $j$ th iteration is to estimate the following two expectations such as,

$$
y_{p}^{(j)}=\hat{\psi}^{(j)}+\beta_{p}\left(y-\sum_{n=1}^{k} \psi_{n}^{(j)}\right)
$$




$$
y_{q}^{(j)}=\sum_{n=1, n \neq k}^{k} \hat{\psi}^{(j)}+\beta_{q}\left(y-\sum_{n=1}^{k} \psi_{n}^{(j)}\right)
$$

$\wedge(j)$

Where $\hat{\psi}$ is the $j^{\text {th }}$ estimate of $\psi^{(j)}$, which represents an unknown parameter vector for the $n^{\text {th }}$ asynchronous user. Furthermore, the non-negative parameters $\beta_{p}$ and $\beta_{q}$ satisfy $\beta_{p}^{2}+\beta_{q}^{2}=1$. Apparently, the expectation step is equal to the Multiple Access Interference (MAI) cancellation procedure. The proposed scheme only evaluates $y_{q}^{(j)}$ because the MAI cancelled signal $y_{p}^{(j)}$ is utilized only by next maximization step.

ii. Maximization Phase : The log-likelihood function can be expressed as

$$
\wedge\left(\tilde{\varepsilon}, \tilde{\mu}, \tilde{t} / y_{p}^{(j)}\right)=\frac{1}{\left(\pi \sigma_{v}^{2}\right)^{2}} \exp \left[-\frac{1}{\sigma_{v}^{2}} \sum_{r=1}^{N_{0}}\left|y_{p}-\tilde{\psi}\right|^{2}\right]
$$

The Channel State Information (CSI) vector for the proposed algorithm is given as

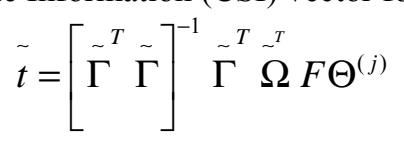

Where,

$$
\Theta^{(j)}=\frac{1}{N_{0}} \sum_{m=1}^{N_{0}} \exp \left[-i \frac{2 \Pi \varepsilon m\left(N+N_{s}\right)}{N}\right] y_{p}^{(j)}
$$

Then the CFO estimate $\hat{\varepsilon}^{(j+1)}$ can be obtained by using $\left\{y_{p}^{(j)} ; 1 \leq m \leq N_{0}\right\}$.

Further, evaluate the timing offset by one dimension search is given as

Where,

$$
\hat{\mu}^{(j+1)}=\arg \max _{0 \leq \tilde{\mu} \leq \mu_{\max }} \tilde{A}^{T} \tilde{V}^{(j)}
$$

$$
\begin{aligned}
& \tilde{t}=\left[\tilde{\tilde{\Gamma}}^{T} \tilde{\Gamma}\right]^{-1} \tilde{\tilde{\Gamma}}^{T} \tilde{\sim}^{T} \boldsymbol{\Omega} F \Theta^{(j)} \\
& \tilde{A}^{(j)}=\tilde{\Omega}^{T} F^{T} \Theta^{(j)}
\end{aligned}
$$

The iteration of the expectation and maximization steps remains until the estimates converge. It is identified that $\beta_{\mathrm{p}}=1$ or $\beta_{\mathrm{q}}=1$ is the optimal condition to maximize the Fisher information of $y_{p}$ and $y_{q}$.

\section{RESULTS AND DISCUSSION}

The Simulation of CRS interference cancellations is performed using Link-level simulations, in order to evaluate the performance of the efficient equalizer and the ESAGE-MAP channel estimation for noncolliding and colliding cases. The simulation parameters for the proposed design are given in Table I where most of the parameters are based on the 3GPP scenarios.

TABLE I : Simulation parameters

\begin{tabular}{|c|c|c|}
\hline Parameters & Serving Cell & Neighbor Cell \\
\hline Bandwidth & $10 \mathrm{MHz}$ & $10 \mathrm{MHz}$ \\
\hline Modulation & $64 \mathrm{QAM}$ & N/A \\
\hline Channel encoder & Turbo Coding & Turbo Coding \\
\hline Channel Estimation & LMMSE & - \\
\hline Cells timing offset & $2.5 \mu \mathrm{s}$ & $2.5 \mu \mathrm{s}$ \\
\hline
\end{tabular}


The strongest interfering cell for colliding CRS and non-colliding CRS cases has signal to noise ratio values $12 \mathrm{~dB}$ and $10 \mathrm{~dB}$ respectively with respect to the serving cell. All cells are synchronized by time, which is that the delays among cells are small $(2.5 \mu \mathrm{s})$ and drops within the cyclic prefix length. The user is able to evaluate the interference power and time delay/offset. In practice, the interference is estimated using Reference Signal Received Power (RSRP) from all cells. The timing delay is found from cell timing information within the cell-search process.

Fig. 3 shows that the Bit Error Rate (BER) performance of the non-colliding CRS-IC for without interference cancellation (No-IC), LLR muting (LLRMut), LMMSE CRS-IC and proposed LS-LMMSE CRSIC.

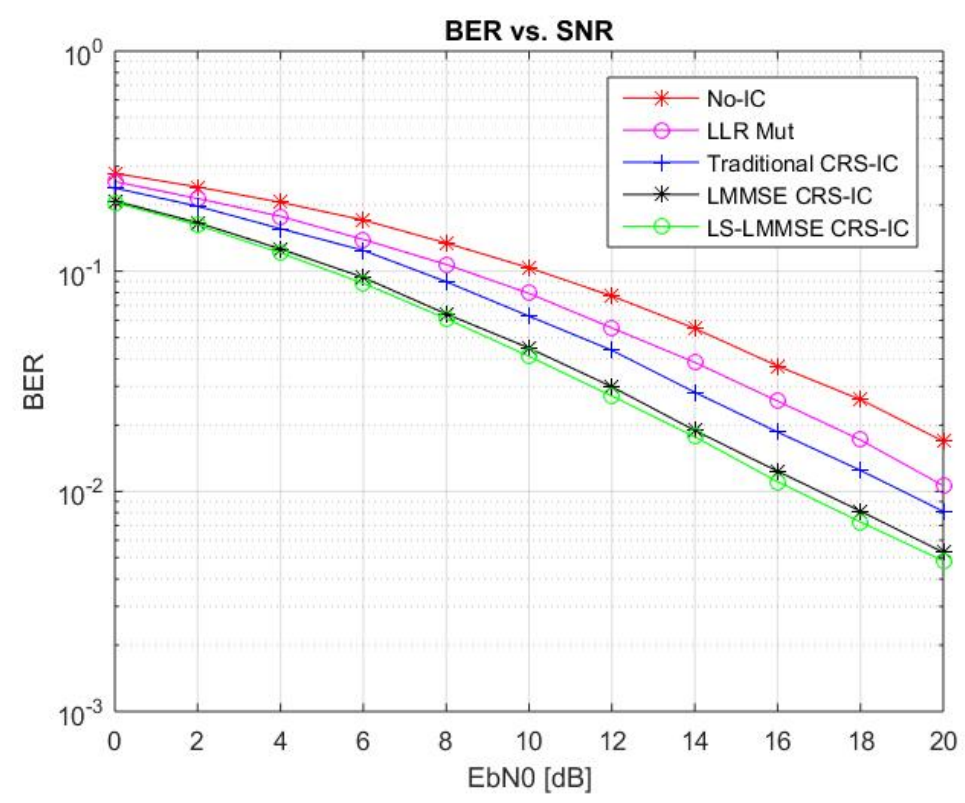

Fig. 3 BER performance for non-colliding CRS.

The non-colliding CRS experiment considered $2 \times 2$ MIMO SFBC with 64 QAM, and Doppler frequency $5 \mathrm{~Hz}$. The exact CRS-IC is represented by ideal CRS-IC which that the interference is ideally cancelled by utilizing the ideal channel estimates of the interfering cell. Furthermore, the perfect CRS-IC can be seen as an upper-bound.

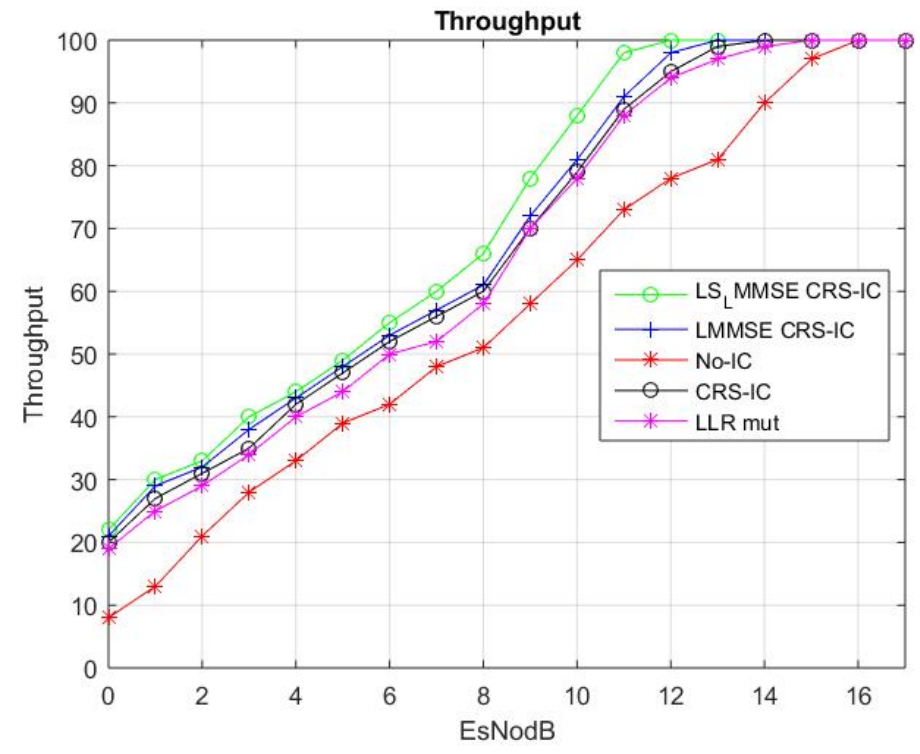

Fig. 4. Throughput performance for non-colliding CRS.

The proposed experimental setup shows the proposed efficient equalizer works very close to the upperbound and ideal CRS-IC. Also, the results are compared with robust equalizer (LMMSE equalizer), LLR muting (LLRMut) are ultra-low complexity receivers for mitigate interference. LLR muting sets the LLR information of 
the interfered symbols to zero prior to data decoding. Fig. 4 shows that proposed results achieved higher throughput when compared to other techniques.

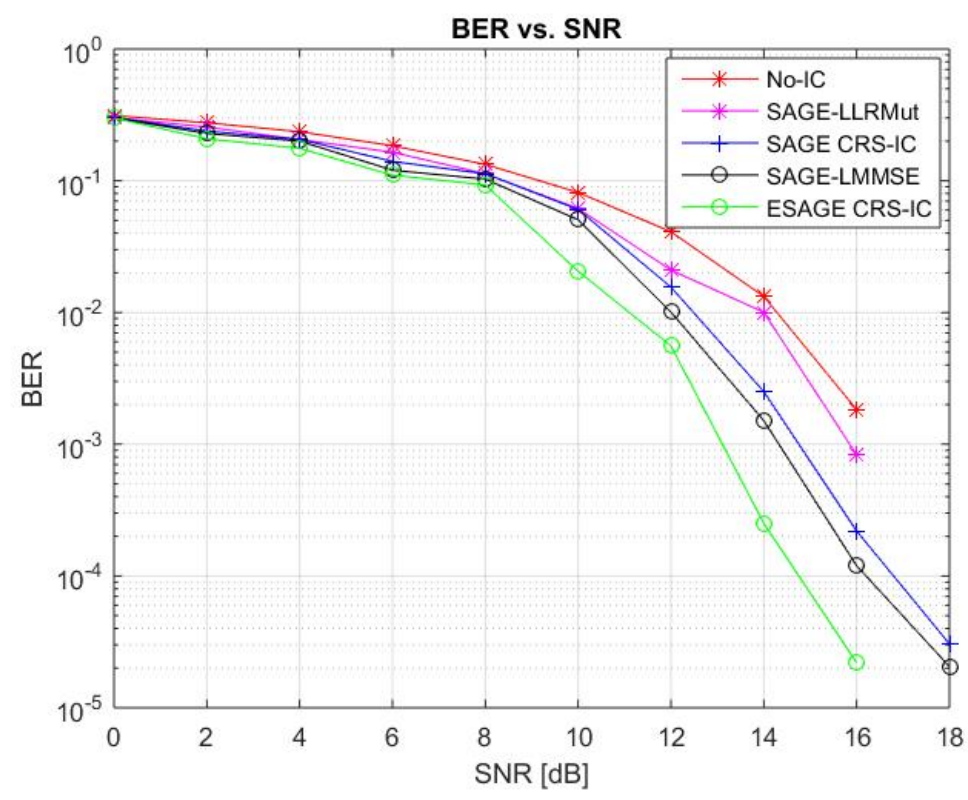

Fig. 5 BER performance for colliding CRS.

Fig. 5 shows that the BER performance of the colliding CRS-IC for without interference cancellation (No-IC), SAGE LLR muting (LLRMut), SAGE LMMSE and proposed ESAGE CRS-IC.

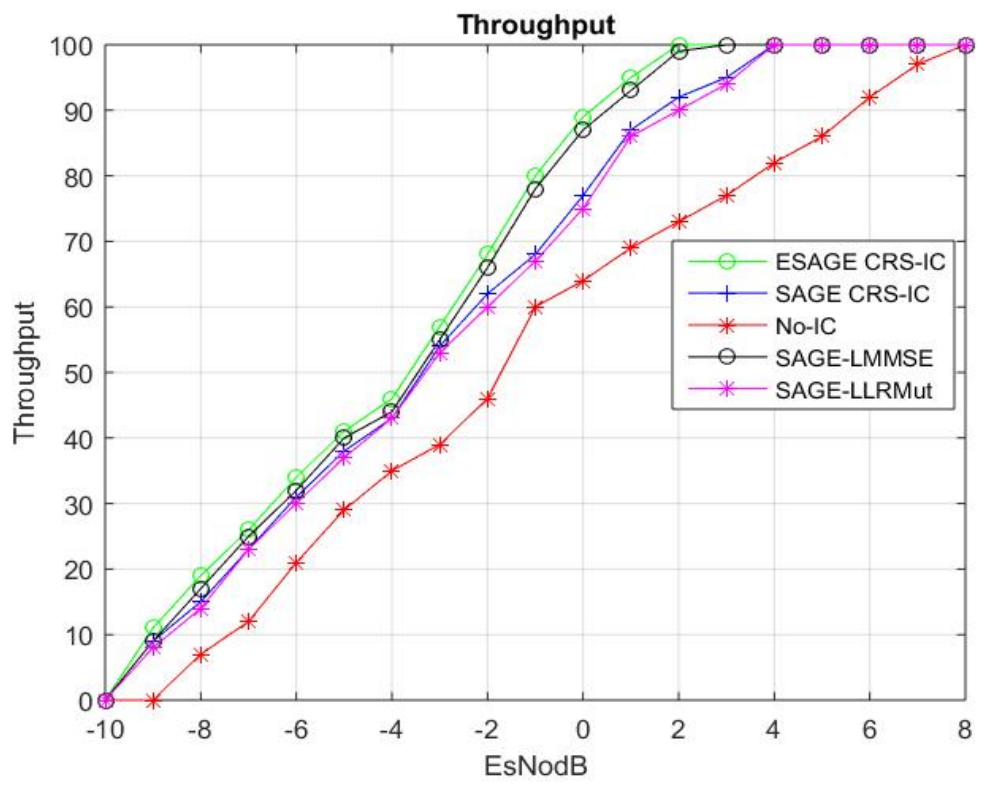

Fig. 6 Throughput performance for colliding CRS.

The simulation results of the colliding CRS scenario use $2 \times 2$ MIMO SFBC 64 QAM with medium correlation channels. The proposed experimental setup shows the proposed ESAGE-MAP work very close to ideal CRS-IC. Also, the results are compared with SAGE-LMMSE, SAGE-LLRmut and SAGE CRS-IC. Fig. 6 shows that the proposed results achieved higher throughput when compared to other techniques. 


\section{CONCLUSION}

In this paper, a user equipment receiver with efficient interference mitigation capabilities is proposed, namely the efficient equalization by grouping the interfered resource elements and performing equalization for the non-colliding CRS. It has high throughput and low BER when compared to without interference cancellation (No-IC), LLR muting (LLRMut), LMMSE CRS-IC and proposed LS-LMMSE CRS-IC. Further, in this paper ESAGE algorithm is investigated for the colliding CRS. The performance has been evaluated and compared with other traditional techniques. Also, the results are compared with SAGE-LMMSE, SAGE-LLRmut and SAGE CRS-IC shown that the proposed results achieved higher throughput when compared to other techniques. The simulation results confirm that the proposed algorithms will provide a better outcome. In future, further improve CRS interference mitigating technique in order to provide high throughput.

\section{REFERENCES}

[1] Simon, R., \&Ahuja, L. (2014). Unravelling Long Term Evolution (LTE) Ownership and Adoption Challenges. Global Journal of Enterprise Information System, 6(2), 48-55.

[2] Agarwal, A., \&Agarwal, K. (2014). The Next Generation Mobile Wireless Cellular Networks-4G and Beyond. American Journal of Electrical and Electronic Engineering, 2(3), 92-97.

[3] Moon, S., Malik, S., Kim, B., Choi, H., Park, S., Kim, C., \& Hwang, I. (2014, January). Cell Range Expansion and Time Partitioning for Enhanced Inter-cell Interference Coordination in Heterogeneous Network. In System Sciences (HICSS), 2014 47th Hawaii International Conference on (pp. 5109-5113). IEEE.

[4] Chiu, C. S., \& Huang, C. C. (2012, May). An interference coordination scheme for picocell range expansion in heterogeneous networks. In Vehicular Technology Conference (VTC Spring), 2012 IEEE 75th (pp. 1-6). IEEE.

[5] Damnjanovic, A., Montojo, J., Wei, Y., Ji, T., Luo, T., Vajapeyam, M. \&Malladi, D. (2011). A survey on 3GPP heterogeneous networks. IEEE Wireless Communications, 18(3).

[6] Tang, W., Zhang, R., Liu, Y., \&Feng, S. (2014, December). Joint resource allocation for eICIC in heterogeneous networks. In Global Communications Conference (GLOBECOM), 2014 IEEE (pp. 2011-2016). IEEE.

[7] Huang, M., \&Xu, W. (2012, April). Macro-femto inter-cell interference mitigation for 3GPP LTE-A downlink. In Wireless Communications and Networking Conference Workshops (WCNCW), 2012 IEEE (pp. 75-80). IEEE.

[8] Shah, B., \&Ghimire, S. (2013). Understanding and Development of Inter-cell Interference Mitigation mechanism in LTE-A Heterogeneous Network.

[9] Davydov, A., \&Morozov, G. (2015, September). Enhanced Interference Cancellation of Cell-Specific Reference Signals for LTE-A. In Vehicular Technology Conference (VTC Fall), 2015 IEEE 82nd (pp. 1-5). IEEE.

[10] Luo, H., Li, W., Zhang, Y., Huang, L. K., Cosmas, J., \& Ni, Q. (2015). CRS interference cancellation algorithm for heterogeneous network. Electronics Letters, 52(1), 77-79.

[11] Soret, B., Wang, Y., \& Pedersen, K. I. (2012, June). CRS interference cancellation in heterogeneous networks for LTE-advanced downlink. In Communications (ICC), 2012 IEEE International Conference on (pp. 6797-6801). IEEE.

[12] Priyanto, B. E., Kant, S., Rusek, F., Hu, S., Chen, J., \&Wugengshi, C. (2013, September). Robust ue receiver with interference cancellation in lte advanced heterogeneous network. In Vehicular Technology Conference (VTC Fall), 2013 IEEE 78th (pp. 1-7). IEEE.

[13] Huang, M., \&Xu, W. (2012, April). Macro-femto inter-cell interference mitigation for 3GPP LTE-A downlink. In Wireless Communications and Networking Conference Workshops (WCNCW), 2012 IEEE (pp. 75-80). IEEE.

[14] Kutz, G., Zach, N., Bar-Or, A., \&Bezalel, K. (2016). LTE Measurements with CRS Interference Cancellation. IEEE Communications Letters, 20(10), 2063-2066.

[15] JIANG Zheng, QIN Xiao-fang, ZHANG Xin, CHANG Yong-yu (2008). SAGE based iterative receiver for joint synchronization and channel estimation in uplink MIMO-OFDMA systems.

[16] Md. Shipon Ali (2015). An Overview on Interference Management in 3GPP LTE-Advanced Heterogeneous Networks.

\section{Author Biography}

S. Shibu received his B.E. Degree in Electronics and Communication Engineering from Manonmaniam Sundaranar University, Thirunelveli, in 2004 and M.E degree in Applied Electronics from Anna University, Chennai, in 2011.He is currently a Research Scholar in Pondicherry Engineering College and working towards his Doctoral degree. His research interests are in the areas of Wireless Communication, Heterogeneous networks, LTE, CRS Interference Cancellation.

V. Saminadan received his BTech and MTech in ECE from Pondicherry Engineering College. He is a Lecturer in the Department of ECE, Pondicherry Engineering College in August 1995. Then he obtained his PhD from Anna University, Chennai in 2006. He has published around 35 papers in national/international conferences/journals. Currently, he is a Professor in the Department of ECE, Pondicherry Engineering College, Puducherry. His areas of interests include optical networks, wireless communication and VLSI design. 